\title{
Proceedings
}

\author{
2017

\section{Network and Distributed System Security Symposium}

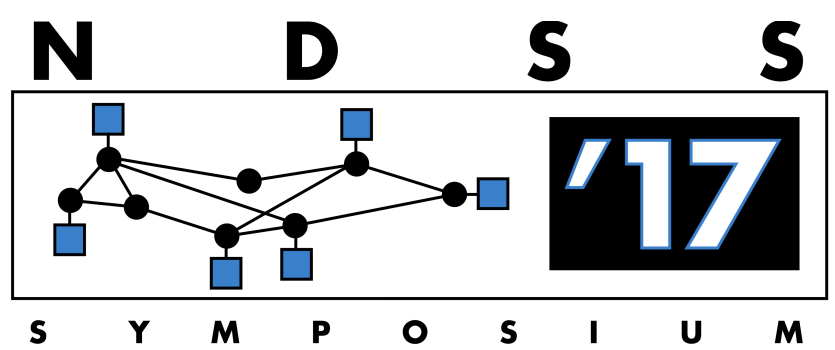




\title{
Proceedings
}

\author{
2017

\section{Network and Distributed System Security Symposium}

\author{
February 26 - March 1, 2017 \\ San Diego, California
}

Sponsored by the

Internet Society

䖯 Internet 


\title{
重耍 Internet
}

Internet Society 1775 Wiehle Avenue Suite 201 Reston, VA 20190-5108

\author{
Copyright @ 2017 by the Internet Society. \\ All rights reserved.
}

Copyright and Reprint Permissions: The Internet Society owns the copyrights for this publication and all of the papers contained herein. Permission to freely reproduce all or part of any paper for noncommercial purposes is granted provided that copies bear the copyright notice and the full citation on the first page. Reproduction for commercial purposes is strictly prohibited without the prior written consent of the Internet Society, the first-named author (for reproduction of an entire paper only), and the author's employer if the paper was prepared within the scope of employment.

Address your correspondence to: Senior Events Manager, Internet Society, 1775 Wiehle Avenue, Suite 201, Reston, Virginia 20190-5108, U.S.A., tel. +1 703439 2120, fax +1 703326 9881, ndss@isoc.org.

The papers included here comprise the proceedings of the meeting mentioned on the cover and title page. They reflect the authors' opinions and, in the interest of timely dissemination, are published as presented and without change. Their inclusion in this publication does not necessarily constitute endorsement by the editors or the Internet Society.

ISBN Number (Digital Format) : 1-891562-46-0

Additional copies may be ordered from:

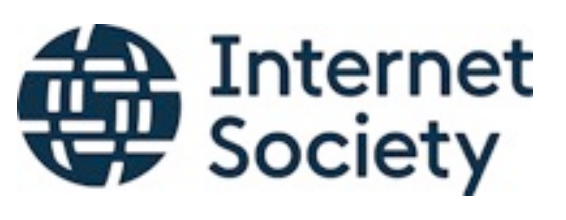

Internet Society

1775 Wiehle Avenue

Suite 201

Reston, VA 20190-5108

tel +1703.439 .2120$

fax +1 703.326.9881

http://www.internetsociety.org 


\title{
Table of Contents
}

\author{
General Chair's Message \\ Program Chair's Message \\ Organizing Committee \\ Program Committee \\ Steering Group
}

Keynote Speaker: J. Alex Halderman, Professor, University of Michigan

\section{Session 1: Applied Crypto and Cryptocurrencies}

IO-DSSE: Scaling Dynamic Searchable Encryption to Millions of Indexes By Improving Locality

I. Miers, P.Mohassel

ObliviSync: Practical Oblivious File Backup and Synchronization A.J. Aviv, S. Geol Choi, T. Mayberry, D.S. Roche

TumbleBit: An Untrusted Bitcoin-Compatible Anonymous Payment Hub E. Heilman, L.Alshenibr, F. Baldimtsi, A. Scafuro, S. Goldberg

P2P Mixing and Unlinkable Bitcoin Transactions

T. Ruffing, P. Moreno-Sanchez, A. Kate

SilentWhispers: Enforcing Security and Privacy in Decentralized Credit Networks G. Malavolta, P. Moreno-Sanchez, A. Kate, M. Maffei

\section{Session 2A: Virtualization and SDN}

DELTA: A Security Assessment Framework for Software-Defined Networks

S. Lee, C. Yoon, C. Lee, S. Shin, V. Yegneswaran, P. Porras

PSI: Precise Security Instrumentation for Enterprise Networks

T. Yu, S. K. Fayaz, M. Collins, V. Sekar, S. Seshan

On the Safety and Efficiency of Virtual Firewall Elasticity Control

J. Deng, H. Li, H.Hu, K.-C. Wang, G.-J. Ahn, Z. Zhao, W. Han

Deconstructing Xen

L. Shi, Y. Wu, Y. Xia, N. Dautenhahn, H. Chen, B. Zang, H. Guan, J. Li 


\section{Session 2B: Web Security}

Thou Shalt Not Depend on Me: Analysing the Use of Outdated JavaScript Libraries on the Web

T. Lauinger, A. Chaabane, S. Arshad, W. Robertson, C. Wilson, E. Kirda

Enabling Reconstruction of Attacks on Users via Efficient Browsing Snapshots

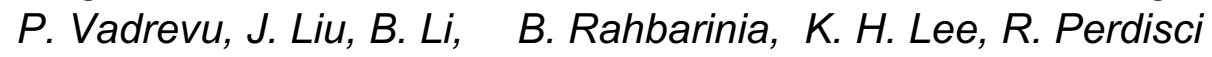

(Cross-)Browser Fingerprinting via OS and Hardware Level Features

Y. Cao, S. Li, E. Wijmans

Fake Co-visitation Injection Attacks to Recommender Systems

G. Yang, N. Z. Gong, Y. Cai

\section{Session 3A: User Authentication}

Broken Hearted: How To Attack ECG Biometrics

S. Eberz, N. Paoletti, M. Roeschlin, A. Patané, M. Kwiatkowska, I. Martinovic

Towards Implicit Visual Memory-Based Authentication

C. Castelluccia, M. Dürmuth, M. Golla, F. Deniz

KEH-Gait: Towards a Mobile Healthcare User Authentication System by Kinetic Energy Harvesting

W. Xu, G. Lan, Q. Lin, S. Khalifa, N. Bergmann, M. Hassan, W. Hu

A Large-scale Analysis of the Mnemonic Password Advice

J. Kiesel, B. Stein, S. Lucks

Cracking Android Pattern Lock in Five Attempts

G. Ye, Z. Tang, D. Fang, X. Chen, K. I. Kim, B. Taylor, Z. Wang

\section{Session 3B: Malware}

Dial One for Scam: A Large-Scale Analysis of Technical Support Scams N. Miramirkhani, O. Starov, N. Nikiforakis

Automated Synthesis of Semantic Malware Signatures using Maximum Satisfiability Y. Feng, O. Bastani

MAMADROID: Detecting Android Malware by Building Markov Chains of Behavioral Models

E. Mariconti, L. Onwuzurike, P. Andriotis, E. De Cristofaro, G. Ross, G. Stringhini

A Broad View of the Ecosystem of Socially Engineered Exploit Documents

S. Le Blond, C. Gilbert, U. Upadhyay, M. Gomez Rodriguez, D. Choffnes 
Catching Worms, Trojan Horses and PUPs: Unsupervised Detection of Silent Delivery Campaigns

B. J. Kwon, V. Srinivas, A. Deshpande, T. Dumitras

\section{Session 4A: TLS et al.}

Measuring small subgroup attacks against Diffie-Hellman

L. Valenta, D. Adrian, A. Sanso, S. Cohney, J. Fried, M. Hastings,

J. A. Halderman, N. Heninger

Indiscreet Logs: Persistent Diffie-Hellman Backdoors in TLS

K. Dorey, N. Chang-Fong, A. Essex

WireGuard: Next Generation Kernal Network Tunnel

J. A. Donenfeld

The Security Impact of HTTPS Interception

Z. Durumeric, Z. Ma, D. Springall, R. Barnes, N. Sullivan, E. Bursztein, M. Bailey

J. A. Halderman, V. Paxson

\section{Session 4B: Secure Computation}

Fast Actively Secure OT Extension for Short Secrets

A. Patra, P. Sarkar, A. Suresh

Constant Round Maliciously Secure 2PC with Function-independent Preprocessing using LEGO

J. B. Nielsen, T. Schneider, R. Trifiletti

Pushing the Communication Barrier in Secure Computation using Lookup Tables

G. Dessouky, F. Koushanfar, A.-R. Sadeghi, T. Schneider, S. Zeitouni,

M. Zohner

Using Fully Homomorphic Encryption for Statistical Analysis of Categorical, Ordinal and Numerical Data W. Lu, S. Kawasaki, J. Sakuma

\section{Session 5A: Mobile Privacy and Security}

Dark Hazard; Learning-based, Large-scale Discovery of Hidden Sensitive Operations in Android Apps

X. Pan, X. Wang, Y. Duan, X.F. Wang, H. Yin

Show Me the Money! Finding Flawed Implementations of Third-party In-app Payment in Android Apps

W. Yang, Y. Zhang, J. Li, H. Liu, Q. Wang, Y. Zhang, D. Gu 
WindowGuard: Systematic Protection of GUI Security in Android

C. Ren, P. Liu, S. Zhu

Obfuscation-Resilient Privacy Leak Detection for Mobile Apps Through Differential Analysis

A. Continella, Y. Fratantonio, M. Lindorfer, A. Puccetti, A. Zand, C. Kruegel, G. Vigna

Automated Analysis of Privacy Requirements for Mobile Apps

S. Zimmeck, Z. Wang, L. Zou, R. lyengar, B. Liu, F. Shaub, S. Wilson, N. Sadeh

S. M. Bellovin, J. Reidenberg

\section{Session 5B: Software and System Security (Part 1)}

Dachshund: Digging for and Securing Against (Non-)Blinded Constants in JIT Code G. Maisuradze, M. Backes, C. Rossow

Safelnit: Comprehensive and Practical Mitigation of Uninitialized Read Vulnerabilities A. Milburn, H. Bos, C. Giuffrida

MARX: Uncovering Class Hierarchies in $\mathrm{C}++$ Programs

A. Pawlowski, M.Contag, V. van der Veen, C. Ouwehand, T. Holz, H. Bos,

E. Athanasopoulos, C. Giuffrida

PT-Rand: Practical Mitigation of Data-only Attacks against Page Tables L. Davi, D. Gens, C. Liebchen, A.-R. Sadeghi

Dynamic Virtual Address Range Adjustment for Intra-Level Privilege Separation on ARM Y. Cho, D. Kwon, H. Yi, Y. Paek

\section{Session 6A: Cloud and Potpourri}

Hello from the Other Side: SSH over Robust Cache Covert Channels in the Cloud

C. Maurice, M. Weber, M. Schwarz, L. Giner, D. Gruss, C. A. Boano,

S. Mangard, K. Römer

Dynamic Differential Location Privacy with Personalized Error Bounds

L. Yu, L. Liu, C. Pu

Are We There Yet? On RPKI's Deployment and Security

Y. Gilad, A. Cohen, A. Herzberg, M. Schapira, H.Shulman

TenantGuard: Scalable Runtime Verification of Cloud-Wide VM-Level Network Isolation

Y. Wang, T. Madi, S. Majumdar, Y. Jarraya, A. Alimohammadifar, M. Pourzandi

L. Wang, M. Debbabi 


\section{Session 6B: Tor}

Dissecting Tor Bridges: A Security Evaluation of their Private and Public Infrastructures S. Matic, C. Troncoso, J. Caballero

The Effect of DNS on Tor's Anonymity

B. Greschbach, T. Pulls, L.M. Roberts, P. Winter, N. Feamster

Avoding The Man on the Wire: Improving Tor's Security with Trust-Aware Path Selection

A. Johnson, R. Jansen, A. D. Jaggard, J. Feigenbaum, P. Syverson

HisTore: Differentially Private and Robust Statistics Collection for Tor

A. Mani, M. Sherr

\section{Session 7: Trusted Execution Environments}

SGX-Shield: Enabling Address Space Layout Randomization for SGX Programs J. Seo, B. Lee, S. Kim. M.-W. Shih, I. Shin, D. Han, T. Kim

T-SGX: Eradicating Controlled-Channel Attacks Against Enclave Programs M.-W. Shih, S. Lee, T. Kim, M. Peinado

BOOMERANG: Exploiting the Semantic Gap in Trusted Execution Environments A. Machiry, E. Gustafson, C. Spensky, C. Salls, N. Stephens, R. Wang,

A. Bianchi, Y. R. Choe, C. Kruegel, G. Vigna

HOP: Hardware makes Obfuscation Practical

K. Nayak, C.W. Fletcher, L. Ren, N. Chandran, S. Lokam, E. Shi, V. Goyal

PANOPLY: Low-TCB Linux Applications With SGX Enclaves

S. Shinde, D. L. Tien, S. Tople, P. Saxena

Keynote Speaker: Trent Adams, Director of Information Security, PayPal

\section{Session 8: Cyberphysical Security}

Hey, My Malware Knows Physics! Attacking PLCs with Physical Model Aware Rootkit

L. Garcia, F. Brasser, M. H. Cintuglu, A.-R. Sadeghi, O. Mohammed,

S.A. Zonouz

ContexloT: Towards Providing Contextual Integrity to Appified loT Platforms Y.J. Jia, Q.A. Chen, S.i Wang, A. Rahmati, E. Fernandes, Z.M. Mao, A. Prakash 
FBS-Radar: Uncovering Fake Base Stations at Scale in the Wild

Z. Li, W. Wang, C. Wilson, J. Chen, C. Qian, T. Jung, L. Zhang, K. Liu,

X. Li, Y. Liu

Internet-scale Probing of CPS: Inference, Characterization and Orchestration Analysis

C. Fachkha, E. Bou-Harb, A. Keliris, N. Memon, M. Ahamad

Wi-Fly? : Detecting Privacy Invasion Attacks by Consumer Drones

S. Birnbach, R. Baker, I. Martinovic

\section{Session 9: Attacks}

ASLR on the Line: Practical Cache Attacks on the MMU

B. Gras, K. Razavi, E. Bosman, H. Box, C. Giuffrida

Unleashing Use-Before-Initialization Vulnerabilities in the Linux Kernel Using Targeted Stack Spraying

K. Lu, M.-T. Walter, D. Pfaff, S. Nürnberger, W. Lee, M. Backes

Address-Oblivious Code Reuse: On the Effectiveness of Leakage-Resilient Diversity R. Rudd, R. Skowyra, D. Bigelow, V. Dedhia, T. Hobson, S. Crane, C. Liebchen, P. Larsen, L. Davi, M. Franz, A.-R. Sadeghi, H. Okhravi

An Evil Copy: How the Loader Betrays You

X. Ge, M. Payer, T. Jaeger

\section{Session 10: Software and System Security (Part II)}

Stack Bounds Protection with Low Fat Pointers

G.J. Duck, R. H. C. Yap, L. Cavallaro

VUzzer: Application-aware Evolutionary Fuzzing

S. Rawat, V. Jain, A. Kumar, L. Cojocar, C. Giuffrida, H. Bos

Self Destructing Exploit Executions via Input Perturbation

Y. Kwon, B. Saltaformaggio, I L. Kim, K. H. Lee, X. Zhang, D. Xu

A Call to ARMs: Understanding the Costs and Benefits of JIT Spraying Mitigations

W. Lian, H. Shacham, S. Savage

Ramblr: Making Reassembly Great Again

R. Wang, Y. Shoshitaishvili, A. Bianchi, A. Machiry, J. Grosen, P. Grosen,

C. Kruegel, G. Vigna 


\title{
General Chair's Message
}

It is my pleasure to welcome you to the 24th Annual Network and Distributed System Security Symposium.

This year we have two collocated workshops: DNS Privacy Workshop and Usable Security (USEC) Workshop. The workshops encompass two critical topics in network and distributed systems security, privacy and usability. I'd like to thank Matthew Smith, the Workshop Chair, and Karen O'Donoghue, Steering Group Co-Chair, for bringing together such an exciting pair of workshops.

Building on recent success, this year we're continuing the tradition of organizing a poster session to showcase both in-progress and exciting recent work in various aspects of computer security. Thanks are due to Johanna Amann and Amir Houmansadr, the Poster Co-Chairs, for making sure we have an excellent poster program.

Due to increased interest in our field and in NDSS, more papers will be presented at NDSS this year than in any past year, yielding a particularly rich and exciting program. Selecting the papers is a task that involves many people and many hours of hard work. l'd like to thank Ari Juels, the Program Chair, as well Patrick Traynor, Shadow Program Chair, for the tremendous amount of work they donated and the excellent job they've done in putting together this year's program.

Many individuals have contributed to making NDSS a success, including everyone on the Steering Group, Organizing Committee, and the Internet Society and Association Management Solutions staff. l'd like thank all of them for contributing their time and effort.

NDSS is possible in large part thanks to our generous sponsors. I'd like to thank Baidu, Cisco, Afilias, Check Point Software Technologies, Qualcomm, San Diego Supercomputer Center, and Salesforce for their support, and the Internet Society for hosting the symposium. Funds for our student grants were provided by the National Science Foundation and the Internet Society.

Finally, thank you, all, for participating in the symposium and through that adding the key ingredient that makes NDSS a success. I wish you all an excellent 24th NDSS!

\author{
Lujo Bauer \\ General Chair, NDSS'17 \\ Carnegie Mellon University
}




\title{
Program Chair's Message
}

It is my great pleasure to welcome you to the 24th Annual Network \& Distributed System Security Symposium (NDSS 2017), held at the Catamaran Resort Hotel and Spa in San Diego, CA, United States from February 26 - March 1, 2017. NDSS fosters information exchange among researchers and practitioners of network and distributed system security. The target audience includes those interested in practical aspects of network and distributed system security, with a focus on actual system design and implementation. A major goal is to encourage and enable the Internet community to apply, deploy, and advance the state of network and distributed systems security technologies.

This year NDSS received a record 423 valid submissions (i.e., not counting papers that clearly violated the submission guidelines). Submissions were evaluated on the basis of their technical quality, novelty, and significance. Papers went through three rounds of review. Reviewing culminated in a one-day in-person program committee meeting, at which 68 papers (approximately 16\%) were selected to appear in the program.

Organizing a conference as large as NDSS is a substantial endeavor, and l'd like to extend my sincere thanks to everyone who contributed her or his time and effort. I'd also like to specifically thank a few individuals who made particular contributions to NDSS 2017. Karen O'Donoghue and Julie Rowland-Lane handled most of the logistics of organizing the conference, as well as shepherding a new program chair. Patrick Traynor served as the shadow chair; my job was made easier by his being there to catch oversights. Yier Jin graciously hosted the PC meeting at the University of Central Florida in Orlando. This year we tried an experiment in which we ran the PC meeting in two parallel tracks. Several students were especially helpful in addressing the logistical challenges this experiment involved. l'd like to thank Fan Zhang for his extensive technical support, Grant Hernandez and Bradley Reaves for their assistance on the ground, and Yan Ji for creating a scheduling tool for the meeting. Thanks also to David Balenson, the Publications Chair, for his efforts and persistence in producing the proceedings you have before you. l'd also like to thank everyone who served on the program committee and put in the time both to review papers and travel to the PC meeting. It was my pleasure and honor to have worked with you to put together the program for NDSS 2017. Also crucial to the success of NDSS are the authors who submitted papers and the attendees, without whom NDSS would not be possible. Welcome to NDSS 2017. I hope you find the program informative and stimulating.

\author{
Ari Juels \\ Cornell Tech \\ Program Chair, NDSS'17
}




\section{Program Committee}

\section{Ari Juels, Cornell Tech (Program Chair) \\ Patrick Traynor, University of Florida (Shadow Program Chair)}

Johanna Amann, ICSI

Manos Antonakakis, Georgia Institute of

Technology

Erman Ayday, Bilkent University

Davide Balzarotti, EURECOM

David Barrera, ETH Zurich

Adam Bates, University of Illinois Urbana

Champaign (UIUC)

Lujo Bauer, Carnegie Mellon University

Alex Biryukov, University of Luxembourg

Kevin Butler, University of Florida

Nicolas Christin, Carnegie Mellon University

Dana Dachman-Soled, University of Maryland

Anupam Datta, Carnegie Mellon University

Emiliano De Cristofaro, University College

London

Tudor Dumitras, University of Maryland

Manuel Egele, Boston University

William Enck, North Carolina State

University

David Evans, University of Virginia

Ittay Eyal, Cornell University

Domenic Forte, University of Florida

Aurelien Francillon, EURECOM

Matthew Fredrikson, Carnegie Mellon

University

Guofei Gu, Texas A\&M University

Alex Halderman, University of Michigan

Amir Herzberg, Bar Ilan University

Amir Houmansadr, University of

Massachusetts Amherst

Yier Jin, UCF

Jaeyeon Jung, Microsoft Research

Yongdae Kim, KAIST

Engin Kirda, Northeastern University

Farinaz Koushanfar, UCSD

Ralf Kuesters, University of Trier

Wenke Lee, Georgia Institute of Technology
Zhenkai Liang, National University of

Singapore

Ben Livshits, Microsoft Research

Long Lu, Stony Brook University

Ivan Martinovic, Oxford University

Jonathan McCune, Google

Patrick McDaniel, Pennsylvania State

University

Sarah Meiklejohn, University College

London

Andrew Miller, UMD / UIUC

Prateek Mittal, Princeton University

Muhammad Naveed, USC

Steven Murdoch, University College London

Steven Myers, Indiana University

Bloomington

Alina Oprea, Northeastern University

Charalampos Papamanthou, University of

Maryland

Chunyi Peng, The Ohio State University

Christina Poepper, New York University Abu

Dhabi

Raluca Popa, University of California,

Berkeley

Mike Reiter, UNC Chapel Hill

Will Robertson, Northeastern University

Ahmad-Reza Sadeghi, TU Darmstadt

Vyas Sekar, CMU

abhi shelat, University of Virginia

Reza Shokri, Cornell Tech

Tom Shrimpton, University of Florida

Ed Suh, Cornell University

Stefano Tessaro, UCSB

Nikos Triandopoulos, Boston University

Venkat Venkatakrishnan, University of

Illinois Chicago

XiaoFeng Wang, Indiana University

Bloomington

Dongyan Xu, Purdue University

Yinqian Zhang, The Ohio State University 


\title{
Organizing Committee
}

\author{
General Chair \\ Lujo Bauer \\ Carnegie Mellon University
}

\section{Program Chair}

Ari Juels

Cornell Tech

\section{Shadow Program Chair}

Patrick Traynor

University of Florida

\section{Local Arrangements Chair \\ Thomas Hutton \\ San Diego Supercomputer Center \\ University of California, San Diego}

\section{Publications Chair}

David Balenson

SRI International

\section{Workshops Chair}

Matthew Smith

Rheinische Friedrich-Wilhelms-Universität Bonn

\section{Poster Co-Chairs}

Johanna Amann

International Computer Science

Institute

\section{Event Manager}

Karen O'Donoghue

Internet Society
Amir Houmansadr

University of Massachusetts Amherst

\section{Event Coordinator}

Julie Rowland

Association Management Solutions 


\title{
Steering Group
}

Co-Chairs

\author{
Lujo Bauer \\ Carnegie Mellon University
}
Karen O'Donoghue
Internet Society

\section{Steering Group Members}

David Balenson

SRI International

Davide Balzarotti

EURECOM Graduate School and

Research Center

Tom Hutton

San Diego Supercomputer Center

Yongdae Kim

Korea Advanced Institute of Science and Technology
Farinaz Koushanfar

University of California San Diego

\author{
Alina Oprea \\ RSA Laboratories \\ Deborah Shands \\ Aerospace Corporation \\ Paul Syverson \\ Naval Research Lab \\ Doug Szajda \\ University of Richmond
}

